\section{The Staining of Polymers}

Ronald W. Smith

2450 Clarke Drive, Lake Havasu City, AZ 86403 orion@uneedspeed.net

In the beginning there was water-the first stain that delineated structure within a polymeric system. The polymer was natural rubber (NR) and the system was an air-dried film of natural latex. Grenquist [1] reported in 1929 that a dried film of natural rubber latex soaked in hot water became milky (Figure 1) and, at that stage, the latex particles could be detected by the optical microscope (OM). The contrast mechanism was provided by the absorption of water by the naturally occurring proteinaceous layers present on the congealed NR particle surfaces. There was little demand for polymer staining until the age of electron microscopy.

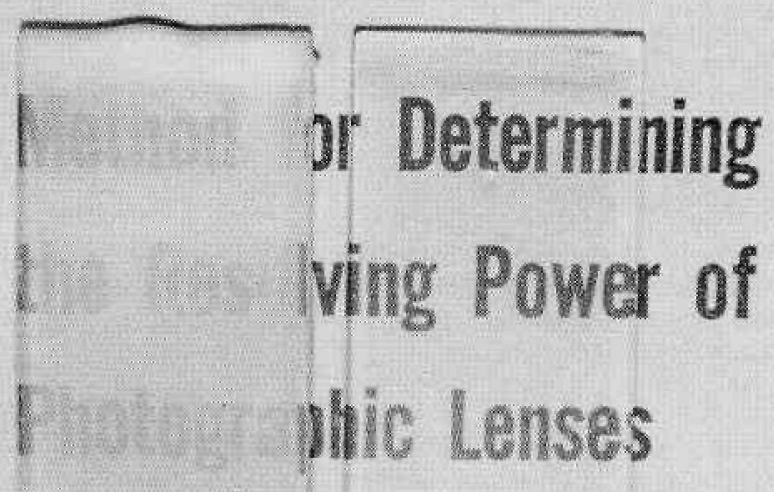

Figure 1: Comparison of Two Natural Rubber Latex Films Left: After Water Immersion, Right: Normal Air-Dried Film

Industrial activity in transmission electron microscopy (TEM) blossomed after WWII and polymer scientists applied this new tool to old problems. It became a valuable tool for studying both natural and synthetic latexes due to the micron and submicron sizes involved. But first, two major problems had to be overcome. The first problem was that NR latex particles (along with some synthetic rubber particles) collapsed on the TEM substrate forming nonspherical blobs. Brown [2] reported that these "soft" particles of unsaturated (containing $>\mathrm{C}=\mathrm{C}<$ ) latexes could be chemically hardened-stained by the chemical process of bromination, thus maintaining their spherical shape and making them perfectly suitable for measurement by electron microscopy. The second problem was that the process of bromination caused an increase in particle diameter! This second problem was addressed for styrene-butadiene copolymers by introducing a "bromination factor" based on the amount of butadiene in the copolymer [3]. Dickenson [4] showed the utility of substituting osmium tetroxide for bromine and later Kato [5] popularized osmium tetroxide staining for acrylonitrile butadiene styrene (ABS) and other similar copolymers. The workhorse of stains has turned out to be ruthenium tetroxide since it works well with either unsaturated polymers [7] or styrene containing polymers [8]. The problem of volume increase caused by staining reactions has received little attention for bulk polymers except for the liquid sulfur hardening (ebonite) method [6] where a volume increase of about 3 percent can be expected.

Ziegler-Natta catalysis opened the floodgates for polymerizing many new polymers and when polymer blending became practiced the demand for new staining methods increased. Examining polymeroriented journals for articles containing micrographs of stained polymeric systems has revealed that since 1929 over 500 articles have appeared. Seventy-one stains for EM, twenty-three stains for $\mathrm{OM}$, and three isotopes for autoradiographical staining have been documented. The major effort is for the staining of bulk polymeric systems where one or more polymers are blended. The text, "Polymer Microscopy" [9], is a basic reference for matching stains with functional groups.

The most distinctive characteristic of bulk polymer staining is that for the pliable unsaturated networks, staining not only highlights target sites but also provides rigidity to the polymer and enables room temperature microtomy. This means two staining protocols exist: 1) staining-hardening of trimmed pyramids in preparation for ultramicrotomy; and 2) staining ultrathin sections or cast thin films.

For bulk staining, the diffusivity of the staining media into the polymer network is critical and the staining media should be tailored for optimum diffusion. It requires several days for aqueous osmium tetroxide to diffuse deeply enough into styrenic matrices (HIPS, ABS) to provide adequate staining-hardening depths, but diethyl ether or tetrahydrofuran (THF) solutions of osmium tetroxide can provide adequate depths in matters of minutes [10]. Epoxy/rubber matrices, especially crosslinked epoxies, require organic solvent media for effective staining. The use of organic solvent media must be carefully monitored to insure that the residence time of the solvent is not so long that it alters morphology. An intermediate between purely aqueous or nonaqueous staining media can sometimes be best. Rubber toughening particles in PVC do not stain well (or at all) with aqueous staining media and organic media tend to overly swell or partially dissolve the PVC matrix. Thus, a suitable intermediate for the PVC/rubber system is a staining medium of $5 \% \mathrm{v} / \mathrm{V}$ THF in water

Bulk staining can present artifacts. Figure 2 is a TEM cross section of poly(styreneacrylonitrile) modified with discrete poly(butadiene) particles that was bulk stained by immersion of a

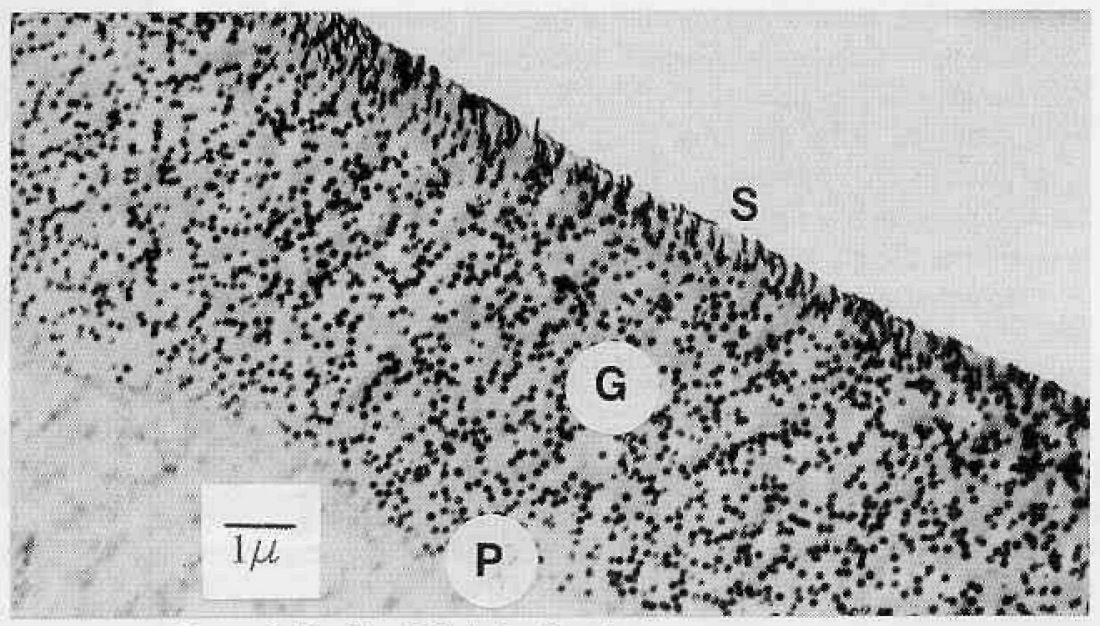

Figure 2: Profile of Staining Depth

ABS Polymer Stained With Aqueous Osmium Tetroxide

trimmed pyramid in $2 \%$ aqueous osmium tetroxide for four days. The stained-hardened layer is five microns deep and presents two cautionary layers. First, is the layer of rubber particle distortion near the surface $S$, where the pyramid was trimmed prior to staining. Microtoming the middle layer, $G$, yields good results. The deepest layer $P_{1}$ between stained and unstained particles, is where partially stained particles appear as donuts and could be 


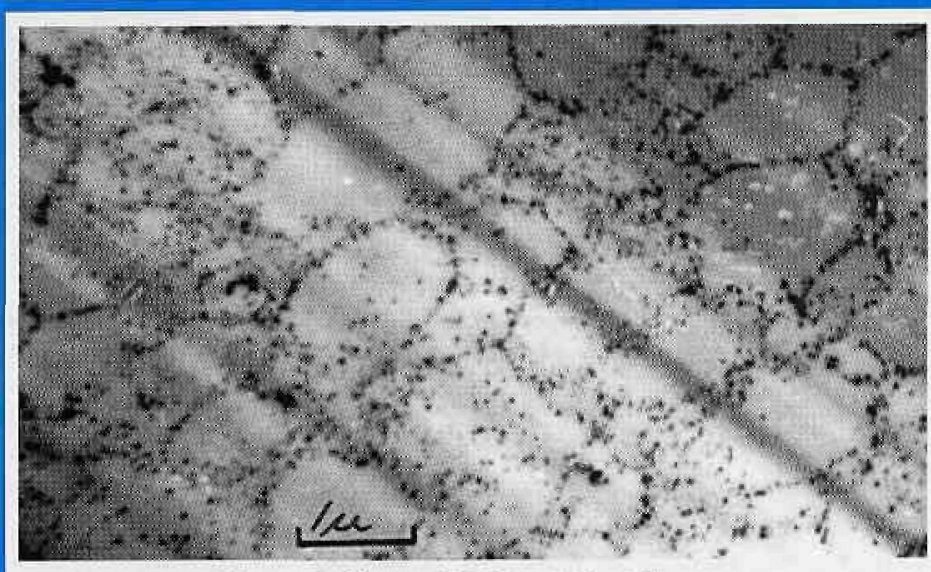

Figure 3: Natural Rubber latex Film

Aqueous Silver Nitrate Stain

wrongly interpreted as core-shell particles. A glance at the unstained matrix shows how effective the staining was for preserving morphology. Aspecial case of bulk staining is the staining of polymer latexes (or dispersions) that is done by adding the staining media to a small volume of diluted latex. Here, it is important to determine the proper dilution of the latex so that particles remain isolated upon drying. Generally, this is in the order of fractional total solids.

Ultrathin sections and cast thin films can be stained using liquids or vapors in a straightforward manner but vapor staining is generally preferred due to ease of handling. Cryo sectioning, if available, can be used to circumvent bulk staining and has the advantage that there is less chance of artifact.

An awareness of what is being stained is essential. Mechanical deformation of some polymer systems produces crazed matter (microfibrils) and stains (silver nitrate [11], liquid sulfur [12] sulfuriodine eutectic [13] and osmium tetroxide [14] can become trapped in the free space between the microfibrils and could be confused with chemical staining. A rate dependant type of staining should be recognized. Amorphous regions in semicrystalline polymers will soak up stains first, leaving the crystalline regions relatively unstained. Differences in staining rate is also a useful tool for differentiating between amorphous polymers. A blend of two amorphous polymers, each with little or no stainable sites, may be differentiated based upon rates of stain perfusion - a phenomenon used to advantage by staining polymer blends at temperatures chosen so that one of them is near or at the glass transition temperature.

Negative staining can be useful other than for outlining particles sitting on flat surfaces. The TEM result shown in Figure 3 for the "water stained" latex film experiment described earlier, was achieved by adding silver nitrate to the hot water [15]. Figure 3 is a microtomed section of NR latex film (sulfur hardened) showing where the water had transported the silver nitrate to the protein layers, staining them, and providing an outline of the original latex particles.

As the number and complexities of polymers has increased (blends, complex copolymers, blocks, grafts, core-shell particles, interpenetrating networks, amphilics, hydrogenated olefinics, etc.), so have the tactics for staining. Chemical alteration of the polymer to make it stainable, such as alkaline hydrolysis to enable osmium staining, has been successful [16]. Most staining with ruthenium tetroxide is via vapor phase but aqueous immersion staining has been introduced [17]. Nonaqueous immersion stains are being used such as ruthenium tetroxide in carbon tetrachloride [18]; phosphotungstic acid in benzyl alcohol [19]; and osmium tetroxide in formaldehyde [20]. Preferential imbibitions of styrene by one polymer of a blend and then staining the styrene with ruthenium tetroxide
[21], introduces a new tactic that could be adapted for other systems. Multiple staining may be required, exemplified by a ruthenium tetroxide-uranyl acetate-lead citrate system [22].

Polymer staining can be challenging and rewarding. The multiplexity of systems is a challenge, and the reward comes when the staining is successful. One must always be reminded that stains are aggressive and environmentally sensitive chemicals. Proper safety and disposal and procedures must be followed.

\section{References}

[1] Grenquist, Rubber Chemistry \& Technol. 2 (1929) 656

[2] Brown, J. of Applied Physics, 18 (1947) 273

[3] Bradford and Vanderhoff, J. Colloid Sci. 14 (1959) 543

[4] Dickenson, "The Chemistry and Physics of Rubber-Like Substances", L. Bateman, Ed. John Wiley \& Sons, New York, 1963 Chapter 3, Biochemistry of Hevea Latex

[5] Kato, Polymer Engineering Sci. 7 (1967) 38

[6] Smith and Andries, Rubber Chemistry \& Technol. 47 (1974) 64

[7] Vitali and Mantani, Polymer, 21 (1980) 1220

[8] Trent et al, J. Polymer Sci. C19 (1981) 315

[9] "Polymer Microscopy", Sawyer and Grubb, 2nd Ed., Chap. \& Hall 1995

[10] Riew and Smith, Journal of Polymer Sci. A9 (1971) 2739

[11] Kambour, Polymer (London) 5 (1964) 143

[12] Kambour, J. Polymer Sci. A7 (1969) 1393

[13] Kambour, Polymer (London) 12 (1973) 237

[14] Borggrene, Polymer 28 (1987) 1499

[15] Smith and Folt, Rubber Chemistry and Technol. 50 (1977) 835

[16] Kanig and Neff, Journal of Colloid and Interface Science 29 (1975) 253

[17] Cao, Polymer Communications, 29 (1988) 66 and 67

[18] Nau, Journal of Material Science, 32 (1997) 5335

[19] Machedo, Journal of Polymer Sci. Part A 37 (1999) 1311

[20] Dutta, Rubber Chemistry and Technol. 65 (1992) 932

[21] Cudby, Journal of Natural Rubber, 12 (1997) 102

[22] Holsti-Miettia, Journal of Polymer Engineering and Sci. 34 395

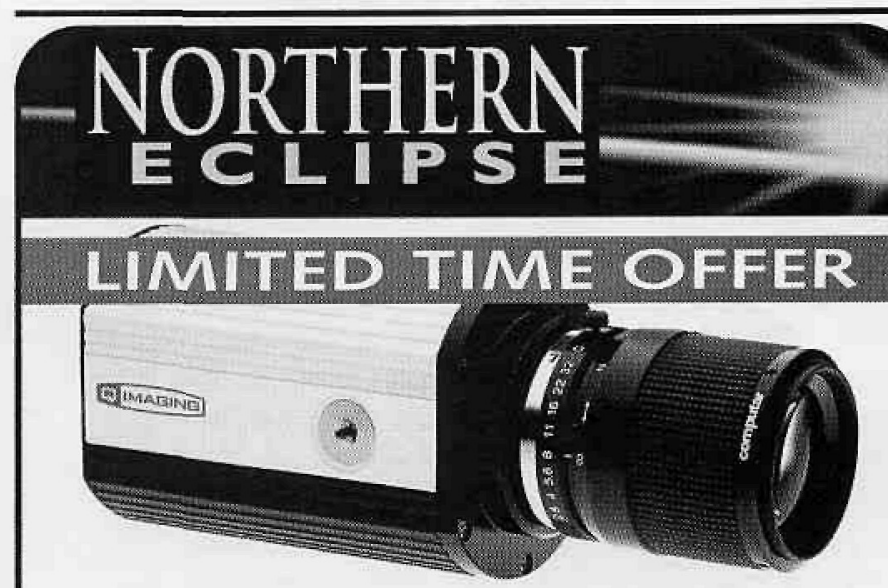

\section{Get the power of Northern Eclipse 6.0 FREE} by ordering a QImaging FireWire CCD camera When you buy a QImaging CCD FireWire camera directly from us at the manufacturer's suggested list price, we will include, for a limited time, one FREE Northern Eclipse 6.0 application of your choice. Applications such as Brightness/ Time, Time-Lapse, Image Merging, Fast Capture to Ram, and even De-convolution are available. See the On Sale page of our web site for a full list of applications available with this offer.

\section{www.empix.com}

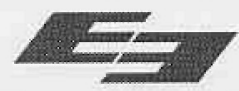

$E M P \mid X$ 


\section{ooming Events}

Society for Neuroscience

November 2-7, 2002, Orlando, FL

www.sfn.org

$\checkmark \quad 28^{\text {th }}$ Intern. Symp. for Testing \& Failure Analysis

November $3-7,2002$, Phoenix, AZ

www.asminternational.org/ISTFA

$\checkmark \quad$ American Vacuum Society

November 4-8, 2002, Denver, Colorado

kahn@ee.princeton.edu

$\checkmark \quad$ Eastern Analytical Symposium

November 18-21, 2002, Somerset, NJ.

WWW: http://www.eas.org/news.html

$\checkmark \quad$ The Materials Research Society

December 2-6, 2002, Fall Meeting, Boston, MA.

info@mrs.org

$\checkmark \quad$ Electron Cryomicroscopy Single Particle Image

Processing and Visualization Workshop

December $11-15,2002$, Houston, TX.

feinberg@bcm.tmc.edu

$\checkmark \quad$ BiOS 2003 Multiphoton Microscopy in the Biomedi cal Sciences III (BO 24)

January 25-31, 2003, San Jose, California

spie@spie.org

$\checkmark \quad$ American Society for Cell Biology

December 14-18, 2002, San Francisco, CA

www.acsb.org

$\checkmark \quad$ Microscopy NZ Conference

February $10-14,2003$, Wellington, NZ

peter.smith@agresearch.co.nz

$\checkmark \quad$ PITTCON 2003

March 9-14, 2003, Orlando, FL

www. pittcon.org

$\checkmark \quad$ Scanning 2003

May 3-5, 2003, San Diego, CA

www.scanning org

$\checkmark$ Microscopical Society of Canada

June 4-6, 2003, Vancouver, BC, Canada

ech@unixg.ubc.ca

$\checkmark \quad$ Microscopy and Microanalysis 2003

August 3-7, 2003, San Antonio, TX

www.msa.microscopy.com

$\checkmark \quad$ American Society for Cell Biology 2003

December 13-17, 2003, San Francisco, CA

www.ascb.org

$\checkmark \quad$ Microscopy and Microanalysis 2004

August 1-5, 2004, Savannah, GA

www.msa.microscopy.com

$\checkmark \quad$ Microscopy and Microanalysis 2005

July 31 - August 4, 2005, Honolulu, HA

www.msa.microscopy.com

Please check the "Calendar of Meetings and Courses" in the MSA journal "Microscopy and Microanalysis" for more details

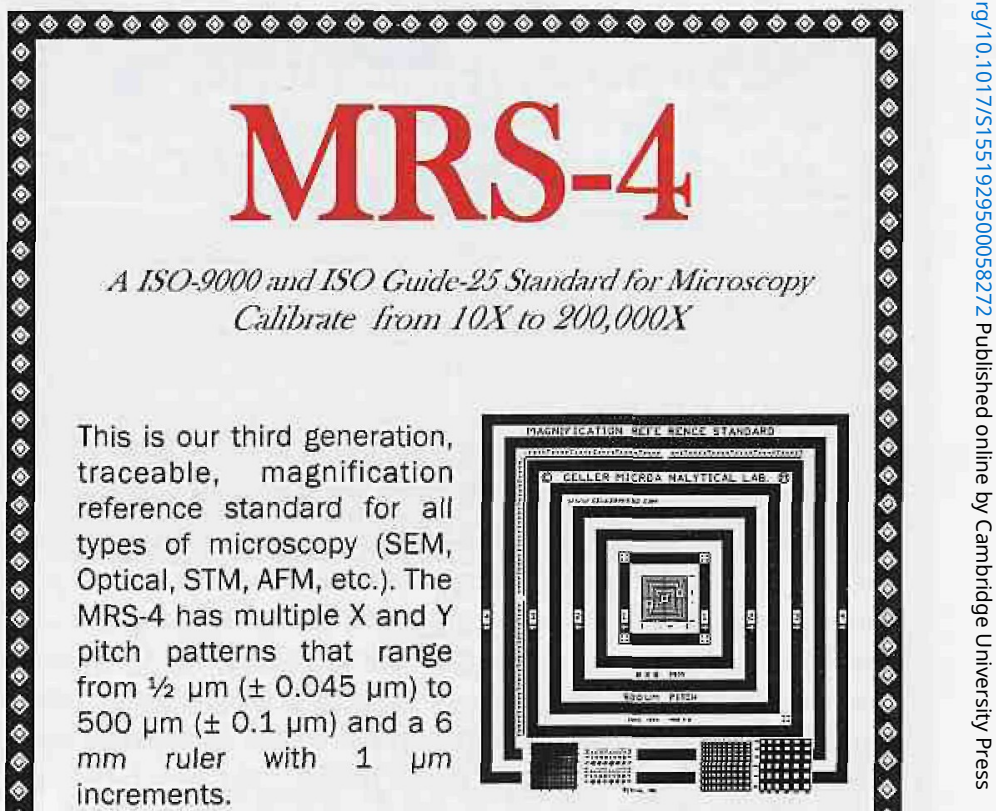

Visit our website and send for our free resources guide.

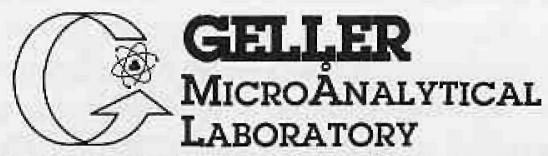

426e BOSTON STREET (RT. 1) * TOPSFIED, MA 01983 978:887-7000 * 9788887-6671 * jg@gellermicro.com http://www.gellermicra.com

\section{Position Available Laboratory Technician}

Applications are invited for the postion of laboratory technician in the field of transmission electron microscopy. The responsibilities include maintenance and operation of the transmission electron microscope and assisting faculty and students in the use of the electron microscope for teaching and research. Appointment will begin from January 1, 2003 or later. Salary is negotiable. The position carries full health and other benefits. Apply to Dr. Eric Hayat, In Charge of the E.M. Facility, Department of Biological Sciences, Kean University, 1000 Morris Ave, Union, NJ. 07083.

e-mail: ehayat@kean.edu 